\title{
A novel endoscopic treatment for renal arteriopelvic fistula post-percutaneous nephrolithotomy (PCNL)
}

\author{
Danny Chao ${ }^{1}$, Alym N. Abdulla ${ }^{3}$, Soojin Kim ${ }^{3}$, Jen Hoogenes², Edward D. Matsumoto³ \\ ${ }^{1}$ Faculty of Medicine, University of Calgary, Alberta; ${ }^{2}$ Department of Surgery and ${ }^{3}$ Division of Urology, \\ McMaster University, Ontario, Canada
}

\section{ABSTRACT}

Main findings: We describe the use of a novel endoscopic approach in the management of unremitting gross hematuria following post-percutaneous nephrolithotomy (PCNL) in a 65-years-old male. This approach proved successful and cost-effective in managing haemorrhage post-PCNL when renal angiography failed to localize the source of bleeding.

Case hypothesis: The recommended treatment modality for renal calculi $\geq 2 \mathrm{~cm}$ is PCNL. It is essential that clinicians are aware of the various complications that can arise from PCNL, including arteriovenous fistula, which is typically managed with renal angio-embolization. The development of a renal arteriopelvic fistula (APF) is an extremely rare complication, and accounts of haemorrhage from renal APF and its treatment have not been well-described in the literature. We successfully hypothesized that the ureteroscopic localization, fulguration, and closure with a fibrin sealant at the site of the arterial bleed results in optimal treatment for this clinical presentation. We report this case in detail.

Promising Future Implications: The successful and cost-effective endoscopic approach described here for treatment of post-PCNL renal APF and unremitting gross hematuria ought to be considered as an adjunct to renal angiography and embolization when the source of bleeding cannot be accurately identified using traditional imaging modalities.

\section{ARTICLE INFO}

\section{Key words:}

Nephrostomy, Percutaneous;

Calculi; Hematuria; Fistula;

Kidney

Int Braz J Urol. 2014; 40: 568-73

Submitted for publication:

October 15, 2013

Accepted after revision:

December 26, 2013

\section{INTRODUCTION}

Percutaneous nephrolithotomy (PCNL) is the recommended treatment modality for renal calculi $\geq 2 \mathrm{~cm}$. In past practice, large renal calculi required open renal surgery (1). Extracorporeal shockwave lithotripsy (ESWL) for large stone burden is not recommended. Retrograde ureteroscopy for large stone burden has a much lower stone-free rate compared to PCNL. Since the advent of endoscopic techniques, the removal of these larger renal calculi has been possible with PCNL, and it seems to offer similar success rates and fewer complication rates when compared to open procedures. Compared to open renal surgery, PCNL is associated with shorter hospital stays and allows the patient an earlier return to work and activities of daily living (2). Furthermore, as PCNL is both less invasive and less expensive to perform than open procedures, it has become the treatment method of choice for large renal calculi.

With the now widespread use of PCNL, it is essential that clinicians are aware of complications that may arise from its implementation. Larger 
stones (e.g. 4-6cm) increase the risk of procedural complications including prolonged operative times and hospital stays, increased risk of infection, and significant blood loss potentially requiring blood transfusions (3). Regardless of stone size, major complications of PCNL include retroperitoneal haemorrhage, sepsis, urinoma, arteriovenous fistula (AVF), pseudoaneurysms, injury to adjacent organs and failure to obtain access. Formation of AVFs following PCNL manifesting as gross hematuria is well described in the literature; these fistulas are typically successfully managed with renal angio-embolization (4). However, formation of a renal arteriopelvic fistula (APF) is rare. This report outlines an experience with a case of renal APF that caused gross unremitting hematuria in a 65-years-old man post-PCNL. We describe a novel endoscopic approach involving ureteroscopic fulguration and ureteroscopic placement of topical fibrin sealant. To our knowledge, renal APFs have not been well described in the literature and this approach has not been previously reported for the treatment of post-PCNL haemorrhage. Previously described as a novel approach for the management of ureteric remnant haemorrhage following laparoscopic radical nephrectomy, this treatment method has significant implications for clinical practice (5). This approach offers a minimally morbid, cost-effective technique for locating and treating the source of bleeding when angio-embolization is unsuccessful.

\section{CASE HYPOTHESIS AND RATIONALE}

A 65-years-old man was treated with PCNL for a left renal $(\geq 2 \mathrm{~cm})$ calculus (Figures $1 \mathrm{~A}, 1 \mathrm{~B}$, and $1 \mathrm{C}$ ) by a community urologist. His medical history was significant for colon cancer, type 2 diabetes mellitus, coronary artery disease, and nephrolithiasis. Two weeks following PCNL, the patient reported an episode of gross hematuria lasting 48 hours that resolved spontaneously. Three weeks later, the patient presented to a community hospital with new onset gross hematuria and passage of clots. He had associated left flank pain, consistent with clot colic. A three-way catheter was inserted and continuous bladder irrigation was initiated. Blood work revealed a normal
Figures $1 \mathrm{~A}, 1 \mathrm{~B}$, and $1 \mathrm{C}$ - Left renal $(\geq 2 \mathrm{~cm})$ calculus.
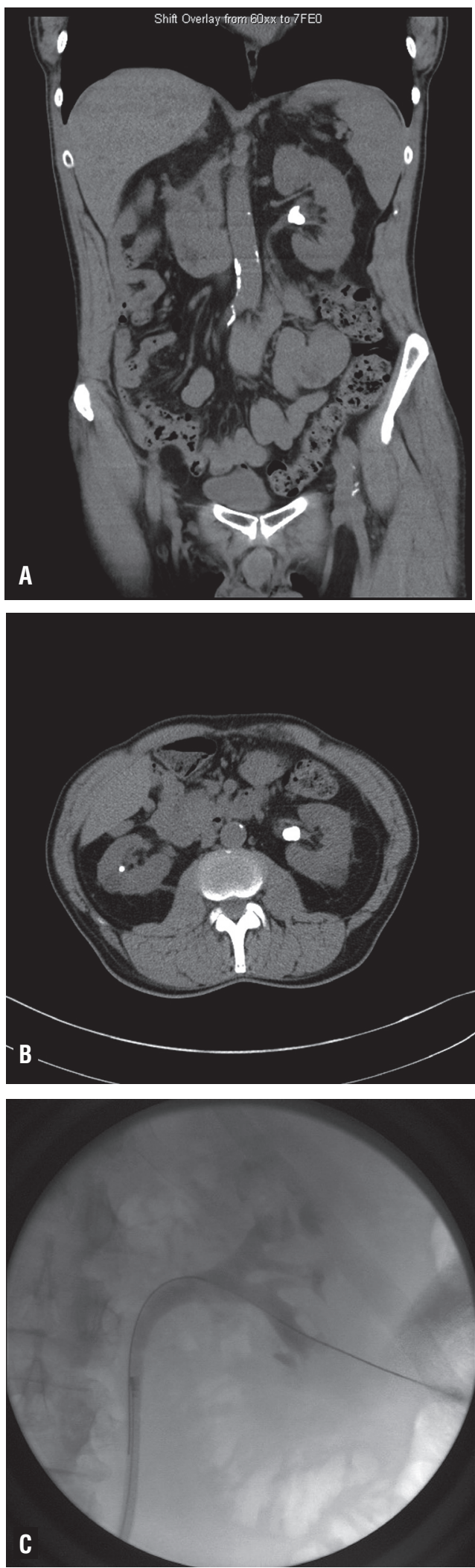
coagulation profile and a haemoglobin level of $10.5 \mathrm{~g} / \mathrm{L}$. An unenhanced computed tomography (CT) scan of the abdomen and pelvis revealed the absence of the large left renal calculus but showed a mild left-sided hydronephrosis with evidence of haemorrhage in the left renal pelvis, left ureter, and bladder. The patient was then transferred to our care at a tertiary academic institution for a renal angiogram and potential embolization.

A renal angiogram was performed but there was no evidence of contrast extravasation. There was also no evidence of a pseudoaneurysm or AVF (Figures 2A and 2B). As a result, no embolization

\section{Figures 2A and 2B - No evidence of a pseudoaneurysm or AVF.}
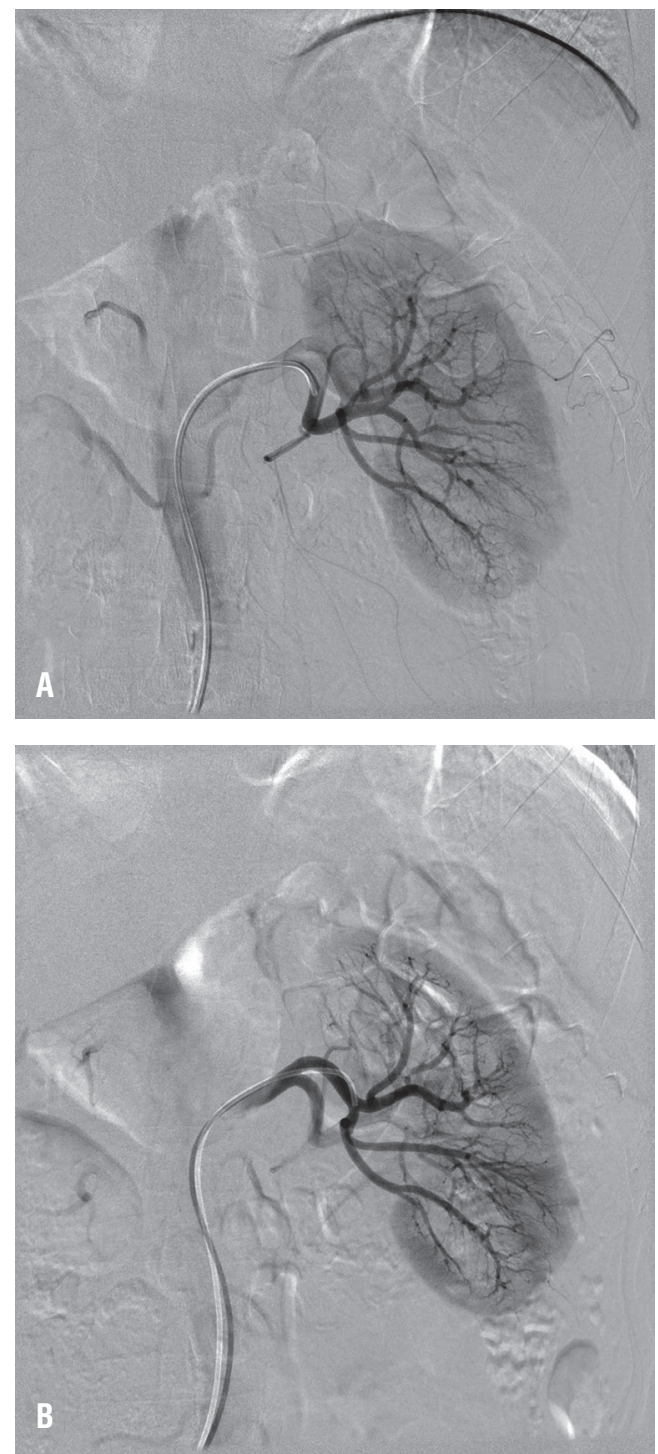

was performed. The following day, the patient's urine was clear but his haemoglobin had declined from $10.5 \mathrm{~g} / \mathrm{L}$ at presentation to $7.9 \mathrm{~g} / \mathrm{L}$ within approximately 36 hours.

The patient was kept in hospital for observation and his hematuria resumed at which point a CT angiogram was obtained. The CT angiogram demonstrated parenchymal changes and blood/ clot in the left kidney and collecting system, consistent with postoperative changes following PCNL (Figures $3 \mathrm{~A}$ and $3 \mathrm{~B}$ ). The scan was again unable to detect any evidence of an AVF. The patient's haemoglobin decreased to $7.0 \mathrm{~g} / \mathrm{L}$ and as a result two

Figures $3 \mathrm{~A}$ and $3 \mathrm{~B}$ - Parenchymal changes and blood/clot in the left kidney and collecting system, consistent with postoperative changes following PCNL.
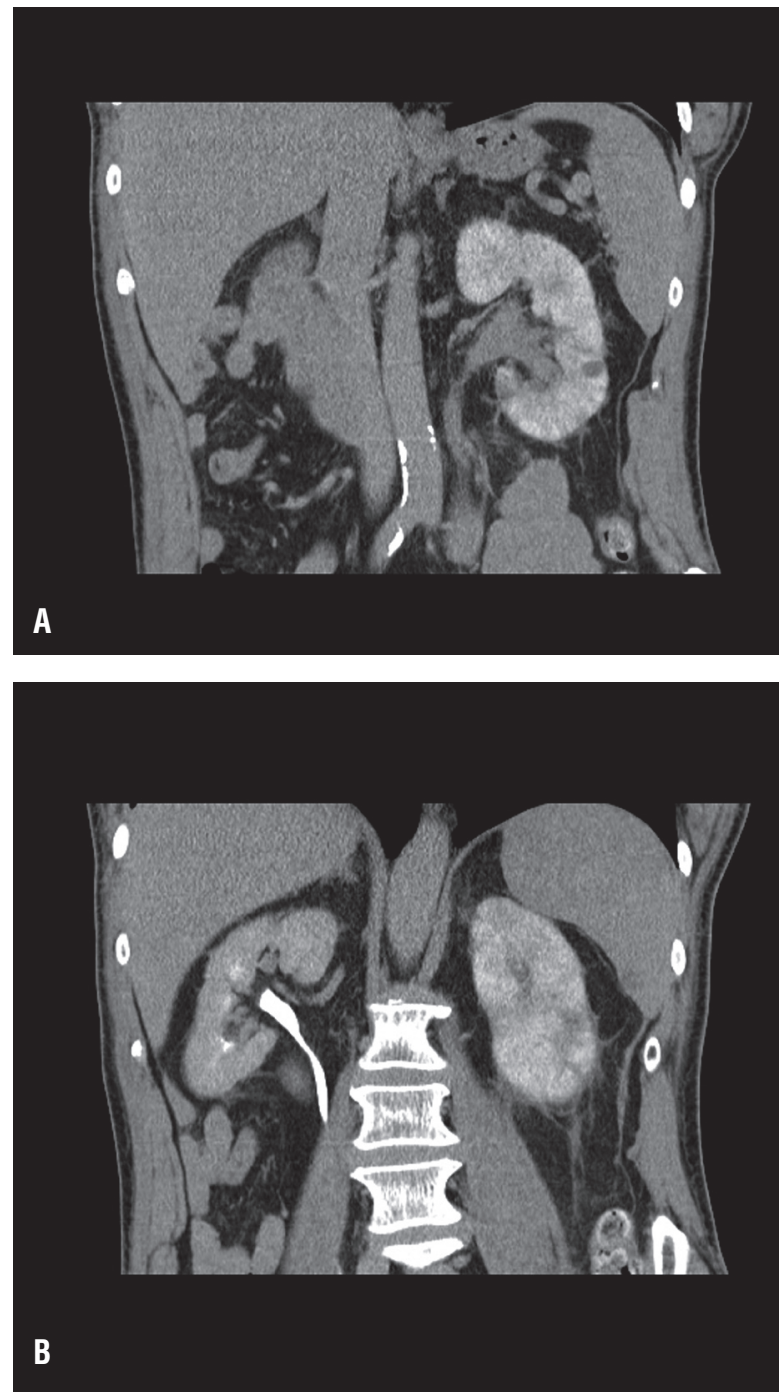
units of blood were transfused. Given the ongoing bleeding and decline in haemoglobin, the patient was taken to the operating room for definitive evaluation and management.

Rigid cystoscopy demonstrated active bleeding from the left ureteric orifice. A retrograde pyelogram was performed which demonstrated filling defects compatible with blood clot. A 5 French angiographic catheter was advanced to the kidney and a formal pyelogram was performed, revealing that the renal pelvis was packed with clot. After introduction of a flexible ureteroscope into the kidney, the hand irrigator (BD Cornwall Syringe System, Mississauga, Ontario, Canada) was used to remove copious amounts of clot. Following complete clot evacuation, an arterial bleed was identified in a lower pole calyx at the site where the original percutaneous access was obtained (Figure-4). The vessel was fulgurated using a Storz-Bugbee electrode through the flexible ureteroscope. A guidewire was placed into the lower pole calyx and an angiographic catheter was positioned (Figure-4). Following this, $5 \mathrm{~mL}$ of the fibrin sealant Tisseel R (Baxter International, Deerfield, IL, USA) was injected via the angiographic catheter to cover the area of fulguration as well as the percutaneous tract. Care was taken to ensure that the Tisseel $\mathrm{R}$ was confined to the lower pole calyx. The flexible uteroscopy was utilized to confirm placement of the Tisseel. The ureteric orifice was subsequently observed for 5 min. at the end of the procedure to ensure adequate hemostasis. The patient was transferred to the ward in stable condition and his hematuria completely resolved. He was discharged home the following day.

\section{DISCUSSION AND FUTURE PERSPECTIVES}

Despite continuous advances in PCNL, bleeding remains an important cause of patient morbidity and requires blood transfusions and renal angio-embolization in a subset of patients. Depending on the stone burden, patient population and comorbidities, post-PCNL transfusion ranges from 8 to 37\% (6). In one series of 582 patients undergoing PCNL, 12\% had bleeding post-operatively which required a blood transfusion (7). In another study with 1338 patients, the rate of post-operative bleeding which required embolization was $0.8 \%$ (8). The rate of post-PCNL bleeding requiring renal angio-embolization throughout the last 20 years has remained consistent between 0.8 to $1.3 \%$ (4). One study reported severe arterial bleeding requiring selective angio-embolization in less than $1.4 \%$ of patients (9). This rate is similar to a recent study of 547 adult patients undergoing PCNL in which 21 patients required a blood transfusion (10). Seven patients required renal angiography and five $(0.9 \%)$ required selective arterial embolization. However, none of these patients presented with hematuria secondary to a

\section{Figure 4 - Arterial bleed was identified in a lower pole calyx at the site where the original percutaneous access was obtained.}

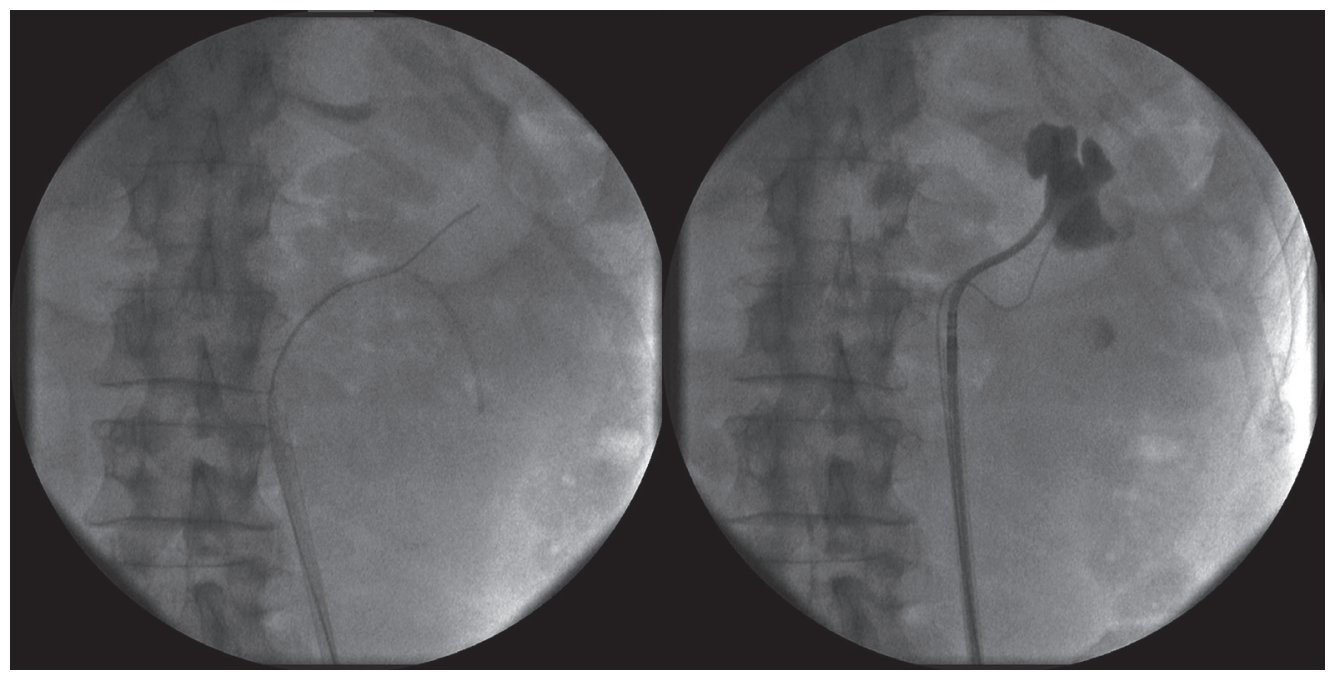


renal APF, highlighting the unique nature of the case presented here.

Renal angiography and embolization is required for both the diagnosis and treatment of arterial haemorrhages, pseudoaneurysms, and AVFs (6). The bleeding location following PCNL has typically been detected with renal angiography. Interestingly, Ueda et al. described two patients with renal haemorrhage in which the site of bleeding could not be detected angiographically (11). Although the two patients underwent percutaneous nephrostomy insertion as opposed to PCNL, the cases are comparable to the present case in that renal haemorrhage developed following a percutaneous intervention and renal angiography was unable to detect the site of haemorrhage. In the cases described by Ueda et al., repeated embolization and irrigations with saline through the nephrostomy catheter following embolization was necessary to stop the bleeding.

The fulguration and fibrin instillation technique for hemostasis described in the current report has been described as a successful option for managing renal haemorrhage. Michael et al. first described this technique in the management of renal haemorrhage from the ureteric remnant following laparoscopic radical nephrectomy in a 61-years-old man (5). Following laparoscopic nephrectomy, bleeding from the ureteric remnant is rare and in the few cases available in the literature, hemostasis is typically obtained through laparoscopic exploration. Similar to the present case, Michael et al. demonstrated successful endoscopic management through ureteroscopic fulguration and instillation of Tisseel.

To our knowledge, accounts of haemorrhage from renal APFs have not been well described in the literature. Renal haemorrhage from AVFs and pseudoaneurysms has been described (6). All previously published accounts have been managed by renal angiography and subsequent embolization. This report describes the use of a novel endoscopic approach to the management of unremitting gross hematuria following PCNL. To our knowledge, this minimally invasive approach has been described in only one case (5). This approach proved successful and cost-effective in managing haemorrhage post-PCNL when renal angiography failed to localize the source of bleeding. While no intra-operative or post-operative difficulties were observed during this case, possible complications specific to the approach described here include failure to identify or control the source of bleeding, ureteropelvic perforation, electrocautery injury to adjacent structures, and infection. As such, post-operative clinical assessments including blood work and imaging to confirm hemostasis should be performed if suspicion exists.

In summary, haemorrhage from renal APFs may occur post-PCNL and it should be considered in the differential diagnosis for patients presenting with unremitting gross hematuria following PCNL. Ureteroscopic localization, fulguration, and closure with a fibrin sealant can be easily attempted at the time of diagnosis. The authors propose that this endoscopic approach be considered as an adjunct to renal angiography and embolization when the source of bleeding cannot be accurately identified using state of the art imaging modalities.

\section{CONFLICT OF INTEREST}

None declared.

\section{REFERENCES}

1. Al-Kohlany KM, Shokeir AA, Mosbah A, Mohsen T, Shoma AM, Eraky I, et al.: Treatment of complete staghorn stones: a prospective randomized comparison of open surgery versus percutaneous nephrolithotomy. J Urol. 2005; 173: 469-73.

2. Preminger GM, Clayman RV, Hardeman SW, Franklin J, Curry T, Peters PC: Percutaneous nephrostolithotomy vs open surgery for renal calculi. A comparative study. JAMA. 1985; 254: 1054-8.

3. Xue W, Pacik D, Boellaard W, Breda A, Botoca M, Rassweiler $\mathrm{J}$, et al.: Management of single large nonstaghorn renal stones in the CROES PCNL global study. J Urol. 2012; 187: 1293-7.

4. Rastinehad AR, Andonian S, Smith AD, Siegel DN: Management of hemorrhagic complications associated with percutaneous nephrolithotomy. J Endourol. 2009; 23: 1763-7.

5. Michael A, Sheridan-Jonah A, Kovac JR, Allard CB, Matsumoto ED: A novel endoscopic treatment for ureteric remnant hemorrhage post laparoscopic radical nephrectomy. Scand J Urol. 2013; 47: 244-7.

6. Lee KL, Stoller ML: Minimizing and managing bleeding after percutaneous nephrolithotomy. Curr Opin Urol. 2007; 17: $120-4$. 
7. Lee WJ, Smith AD, Cubelli V, Badlani GH, Lewin B, Vernace F, et al.: Complications of percutaneous nephrolithotomy. AJR Am J Roentgenol. 1987; 148: 177-80.

8. Duvdevani M, Razvi H, Sofer M, Beiko DT, Nott L, Chew $\mathrm{BH}$, et al.: Third prize: contemporary percutaneous nephrolithotripsy: 1585 procedures in 1338 consecutive patients. J Endourol. 2007; 21: 824-9.

9. Srivastava A, Singh KJ, Suri A, Dubey D, Kumar A, Kapoor $R$, et al.: Vascular complications after percutaneous nephrolithotomy: are there any predictive factors? Urology. 2005; 66: 38-40.

10. Keoghane SR, Cetti RJ, Rogers AE, Walmsley BH: Blood transfusion, embolisation and nephrectomy after percutaneous nephrolithotomy (PCNL). BJU Int. 2013; 111 628-32.
11. Ueda J, Furukawa T, Takahashi S, Miyake O, Itatani H, Araki $Y$ : Arterial embolization to control renal hemorrhage in patients with percutaneous nephrostomy. Abdom Imaging. 1996; 21: 361-3.

Correspondence address:

Edward D. Matsumoto, MD

McMaster Institute of Urology

St. Joseph's Healthcare Hamilton 50 Charlton Ave. East

Hamilton, ON, L8N 4A6, Canada Fax: +1 905 308-7205

E-mail: matsumo@mcmaster.ca 